\title{
Calidad educativa de los centros de ideario católico. Indicadores para su evaluación
}

\section{Educational quality of the centers of catholic ideology. Indica- tors for its evaluation}

INMACULADA DÍAZ-CLAUDEL

RR. EE. Pías de la Imnmaculada FDSM. Córdoba.

(D) https://orcid.org/0000-0002-7547-0424

Ma AMOR MARTÍN-FERNÁNDEZ

Centro de Magisterio Sagrado Corazón, adscrito a la Universidad de Córdoba.

(D) https://orcid.org/0000-0002-5744-6287)

IGNACIO GONZALEZ-LÓPEZ

Departamento de Educación. Universidad de Córdoba.

(D) https://orcid.org/0000-0002-9174-4370

\section{RESUMEN}

Las instituciones educativas necesitan atender a las demandas planteadas por la sociedad. Concretamente los centros de ideario católico, en su constante búsqueda de la excelencia educativa, la satisfacción de las familias y la mejora continua, deben establecer un sistema que gestione eficazmente todos los ámbitos de la organización y garantice su calidad. En este artículo se muestra el análisis bibliográfico realizado en torno a modelos de gestión de la calidad, que han destacado por sus resultados en el ámbito empresarial y sus adaptaciones al ámbito educativo. Se han analizado y combinado sus principios fundamentales para obtener unas dimensiones e indicadores con los que elaborar un modelo final aplicable a estos centros educativos. Con un proceso sistemático de análisis sobre los planteamientos de un estudio descriptivo-comparativo, se descubre la importancia del liderazgo, la organización y gestión de los procesos, y la formación de los profesionales que participan en el proceso. El modelo de gestión de la calidad, que se propone, está orientado concretamente a los centros concertados de ideario católico que imparten enseñanzas en las etapas de Educación Infantil, Educación Primaria y Educación Secundaria, llamados a responder, con un proyecto propio, a su ideario y misión.

\section{ABSTRACT}

Educational institutions need to meet the demands posed by society. Specifically, the Catholic ideology centers, in their constant search for educational excellence, family satisfaction and continuous improvement, must establish a system that effectively manages all areas of the organization and guarantees its quality. This article shows the bibliographic analysis carried out around quality management models, which have stood out for their results in the business field and their adaptations to the educational field. Its fundamental principles have been analyzed and combined to obtain dimensions and indicators with which to develop a final model applicable to these educational centers. With a systematic process of analysis on the approaches of a descriptivecomparative study, the importance of leadership, organization and process management, and the training of professionals involved in the process is discovered. The quality management model, which is proposed, is specifically oriented to the centers of Catholic ideology that teach in the stages of Pre-school Education, Primary Education and Secondary Education, called to respond, with a project of their own, to their ideology and mission.

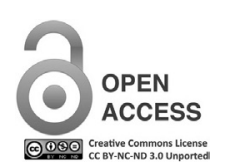

Para citar este artículo: Díaz-Claudel, I., Martín-Fernández, M.A. y González-López, I. (2019). Calidad educativa de los centros de ideario católico. Indicadores para su evaluación. EA, Escuela Abierta, 22, 47-68. doi:10.29257/EA22.2019.06
Recibido: 09/05/19

Aceptado: 26/09/19

PALABRAS CLAVES

Calidad de la educación, educación, evaluación, investigación educativa, gestión y modelos, educación católica

\section{KEYWORDS}

Quality of Education, Education, Evaluation, Educational Research, Management and Models, Catholic Education. 


\section{SISTEMAS Y HERRAMIENTAS PARA LA EVALUACIÓN DE LA CALIDAD DE LOS CENTROS EDUCATIVOS}

El desarrollo de sistemas de evaluación de la calidad educativa se sustenta en una visión determinada del concepto de calidad educativa. Preguntarse por la inclusión de la calidad en educación responde a la obligación de las instituciones educativas por atender a las demandas de excelencia planteadas por la sociedad. La educación no es el único ámbito sometido a evaluación, sino que todas y cada una de las prestaciones sociales han de responder a unos criterios de calidad deseables.

Desde el punto de vista de Pérez, López, Peralta, y Municio (2000), el concepto de calidad aplicado a la educación tiene un claro componente económico, que incluye la existencia de recursos, su utilización y los resultados conseguidos. Al mismo tiempo, se trata de un concepto relacionado con la excelencia en los conocimientos a transmitir, el grado de implicación de la institución ante las necesidades y demandas de la sociedad, la capacidad de organización reflejada en sus actividades y resultados, así como la satisfacción de todas las personas implicadas. Estas dimensiones responden a la perspectiva del Modelo Europeo de Gestión de la Calidad (MEC, 1997), que considera a la institución como un sistema en el cual sus diferentes elementos están relacionados entre sí y todos han de ser el objeto de evaluación y mejora continua.

El proceso de incorporación de un sistema de gestión de calidad en un centro se desarrolla a través de diversas fases, concretadas por Rey y Santa María (2000) en tres: «en primer lugar, se parte del diseño del servicio educativo que se desea prestar; a continuación, se ha de establecer el modelo de organización adecuada en consonancia con el servicio diseñado para, finalmente, realizar una gestión de calidad eficaz» (p. 32).

Por lo tanto, la implantación de un modelo de evaluación de la calidad en una organización educativa se fundamenta en principios de participación, orientación, satisfacción y mejora permanente. A estos hay que añadir una serie de principios operativos que garanticen el desarrollo del mismo. Entre otros, no deben faltar los principios de compromiso y dirección del equipo directivo de la organización, de capacidad de trabajo en equipo eficaz e intencional, de participación de todos los miembros en todos los niveles y, finalmente, de resolución sistemática de problemas (González, 2000).

Existen diversos modelos que, previa adaptación, pueden utilizarse en el ámbito educativo, como son el modelo Deming (1951), el modelo Calidad Total, TQM (1950-1960), el modelo Malcolm Baldrige (1987), el Modelo Europeo de Gestión de la Calidad, EFQM (1992) y la familia de normas de estandarización y acreditación ISO 9001 (2015).

Entre los modelos citados, que derivan en sistemas de gestión de la calidad aplicables en centros educativos, se encuentra el modelo Deming (1951), que ejerce una gran influencia en el desarrollo del control y gestión de la calidad en Japón. Su objetivo básico fue convertirse en una herramienta para mejorar y transformar la gestión de las organizaciones japonesas. Actualmente, se implanta en aquellas empresas que contribuyen de manera muy significativa al desarrollo de la dirección y control de calidad, y supone un acicate para promover la gestión de la calidad en numerosas compañías que encuentran en él una excelente ocasión para comenzar a aprender.

Un segundo modelo es el denominado Calidad Total - TQM (1950-1960), consistente en aplicar el concepto de Calidad Total a los sistemas de gestión de la empresa. Pretende integrar la calidad en todos los procesos de la organización. La implantación de un sistema TQM sirve para ayudar a la organización a conseguir el máximo de eficiencia y flexibilidad en todos sus procesos, enfocándola hacia la obtención de los objetivos a 
corto y medio plazo. Este modelo, aún siendo de la década de los años cincuenta, sigue resultando actual, ya que sus objetivos se orientan a conseguir la satisfacción global, indicador requerido prioritariamente por las empresas e instituciones de hoy.

El modelo Malcolm Baldrige (1987) es una herramienta para la evaluación, mejora y planificación hacia la gestión de la excelencia. Este modelo establece que los líderes de la organización deben estar orientados a la dirección estratégica y a los clientes. También deben dirigir, responder y gestionar el desempeño basándose en los resultados. Las medidas y los indicadores del desempeño y el conocimiento organizativo serán la base sobre la que construir las estrategias clave, que se relacionan con los procesos y con la alineación de los recursos. De este modo se conseguirá una mejora en el desempeño general de la organización y en la satisfacción de los consumidores y de los grupos de interés. Este modelo es en la actualidad marco de referencia para la evaluación del premio nacional de calidad de Estados Unidos. Hertz (2019), afirma que los Criterios Baldrige proporcionan una manera integral para lograr y mantener un alto rendimiento en todas las organizaciones. El Instituto Nacional de Estadística NIST (2011), afirma que los Criterios Baldrige proporcionan una manera integral para lograr y mantener un alto rendimiento en todas las organizaciones.

El modelo Europeo de Gestión de la Calidad EFQM (1988) ofrece una herramienta integral con el objetivo de ayudar a las organizaciones a conocerse mejor a sí mismas, a realizar un análisis objetivo, riguroso y estructurado de su funcionamiento y, en consecuencia, a mejorar su gestión. Se puede decir que el Modelo EFQM es un diagrama de causa y efecto. Si se quiere lograr un resultado diferente, se necesita cambiar algo de lo que se hace dentro de la organización. Su sistema ayuda a identificar puntos fuertes y oportunidades de mejora, establece un nivel de excelencia en gestión en cada uno de los aspectos clave y establece prioridades sobre las que actuar.

Otro modelo destacable se corresponde con la familia de normas de estandarización y acreditación ISO 9001 (2015). Esta se puede aplicar en cualquier tipo de organización o actividad orientada a la producción de bienes o servicios. Recoge tanto el contenido mínimo, las guías y las herramientas específicas de implantación, como los métodos de auditoría con los que periódicamente se analiza el estado de la calidad en la gestión de la institución.

Finalmente, un sistema de calidad ha de responder a la misión, visión y objetivos de la institución a la que vaya dirigido. Por ello, se ha analizado y tomado como referente el Instrumentum laboris (en adelante I.L.) "Educar hoy y mañana. Una pasión que se renueva” de la Congregación para la Educación Católica (2014), como herramienta que define las características fundamentales de las escuelas y de las universidades católicas, y los desafíos a los cuales están llamadas a responder con un proyecto propio.

\section{OBJETIVOS}

Este trabajo se enmarca en una investigación más amplia sobre la evaluación de la calidad educativa de los centros educativos de ideario católico y tiene como finalidad elaborar un modelo de indicadores que permita mantener una gestión de la calidad actualizada, proporcionando a los centros de ideario católico un análisis fiable y válido que les permita adoptar una toma de decisiones para la mejora de sus servicios, derivada de las evaluaciones y lo más acertada posible. Específicamente, este trabajo persigue los siguientes objetivos:

- Seleccionar los modelos de gestión de la calidad empleados en diferentes instituciones educativas. 
- Analizar y discriminar los principios, dimensiones e indicadores que sirven de base para la gestión de la calidad de instituciones educativas en los modelos anteriormente seleccionados.

- Reconocer y determinar los elementos comunes de los diferentes modelos de gestión de calidad y que sirvan de base para la creación de un modelo adaptado a centros educativos de ideario católico.

- Incluir los principios del documento "Educar hoy y mañana. Una pasión que se renueva" (Congregación para la Educación Católica, 2014) en el modelo de gestión de calidad propuesto.

\section{MATERIAL Y MÉTODOS}

Para dar respuesta a las metas de este estudio se ha seguido un proceso sistemático de análisis sobre los planteamientos de un estudio documental descriptivo-comparativo. Se trata de una actividad sistemática y planificada que supone examinar documentos escritos cuya pretensión, en palabras de Del Rincón, Arnal y Latorre (1995), es "obtener información útil y necesaria para dar respuesta a los objetivos identificados en el planteamiento de la investigación" (p. 341). Se entiende como un estudio del material bibliográfico que existe en el área de interés para la investigación (Eyssautier, 2006).

Los modelos de gestión de calidad revisados y seleccionados son: el modelo Deming, el modelo Calidad Total TQM, el modelo Malcolm Baldrige, el Modelo Europeo de Gestión de Calidad EFQM y la familia de normas ISO, actualmente en vigor (versión 9001:2015). Su selección se ha llevado a cabo atendiendo a su adaptación al ámbito educativo y a la valoración sobre ellos de los autores de referencia: Gento (1996), Walton (2004) y Charantimath (2011) para el modelo Deming; Eduardo y Espinoza (2008) y Hasan (2013) para el modelo Calidad Total TQM; Andrade y Labarca (2001), Membrado (2002) y Valenzuela y Rosas (2006) para el modelo Malcolm Baldrige; Maderuelo (2002), Municio (2004), Domínguez y Lozano (2005), Martínez y Riopérez (2005) y Camisón et al. (2006) en referencia al Modelo Europeo de Gestión de Calidad EFQM, y, por último Cidad (2004), Del Río (2008) y Gaviria y Dovale (2014) para la familia de normas ISO. A estos modelos se unen los principios del I. L. (Congregación para la Educación Católica, 2014) para atender a las necesidades del ideario, misión y visión de un centro educativo de ideario católico.

El procedimiento analítico se ha configurado en tres fases, con el fin de diseñar el modelo de gestión de calidad objeto de este trabajo:

FASE 1. Comparación de modelos de gestión de calidad en atención a finalidades y dimensiones.

FASE 2. Análisis, identificación e incorporación de los fines y elementos definitorios de la educación católica, para el desarrollo de la fase 3.

FASE 3. Elaboración de un modelo propio de indicadores de gestión de la calidad que atienda al encargo recibido por los centros de ideario católico. 


\section{RESULTADOS OBTENIDOS}

\subsection{Análisis comparativo de los modelos de gestión de la calidad seleccionados}

El estudio comienza con el análisis comparativo de los modelos de gestión de la calidad seleccionados, que presta especial atención a las finalidades perseguidas, a sus dimensiones constitutivas y a los indicadores que las definen.

En el análisis, llama la atención la diferencia en el número de principios en cada uno de ellos (ver Tabla 1). Deming cuenta con 14 principios, Calidad Total 6, Baldrige 11, EFQM cuenta con 8 y la norma ISO 9001:2015 con 7. Resulta significativo comparar los principios del EFQM con los de Baldrige, pues en los 8 primeros, referidos a la orientación a resultados, adopción de la nueva filosofía, liderazgo, enfoque directivo, personal, aprendizaje e innovación, alianzas y responsabilidad social, apenas existen diferencias, aunque sí en los 3 últimos, que son totalmente nuevos para el Modelo EFQM (ver Tabla 1).

\section{Tabla 1}

Principios fundamentales de los modelos de gestión de la calidad seleccionados

\begin{tabular}{|c|c|c|c|c|c|}
\hline ASPECTO ANALIZADO & Deming & Calidad Total & BALDRIGE & EFQM & ISO 9001:2015 \\
\hline $\begin{array}{l}\text { Servicio de calidad desde } \\
\text { la perspectiva cristiana }\end{array}$ & $\begin{array}{l}\text { Crear y difundir } \\
\text { visión, propósito, } \\
\text { misión }\end{array}$ & $\begin{array}{l}\text { Mejoramiento ha- } \\
\text { cia la calidad total }\end{array}$ & $\begin{array}{l}\text { Enfoque en los } \\
\text { resultados y en la } \\
\text { creación de valor }\end{array}$ & $\begin{array}{l}\text { Orientación en } \\
\text { los resultados }\end{array}$ & $\begin{array}{l}\text { Enfoque al clien- } \\
\text { te }\end{array}$ \\
\hline $\begin{array}{l}\text { Unidad en la misión, vi- } \\
\text { sión y valores }\end{array}$ & $\begin{array}{l}\text { Aprender y adop- } \\
\text { tar la nueva filo- } \\
\text { sofía }\end{array}$ & $\begin{array}{l}\text { Aprender y adop- } \\
\text { tar la nueva filo- } \\
\text { sofía }\end{array}$ & $\begin{array}{l}\text { Aprender y adop- } \\
\text { tar la nueva filo- } \\
\text { sofía }\end{array}$ & $\begin{array}{l}\text { Aprender y } \\
\text { adoptar la nueva } \\
\text { filosofía }\end{array}$ & $\begin{array}{l}\text { Aprender y adop- } \\
\text { tar la nueva filo- } \\
\text { sofía }\end{array}$ \\
\hline Autonomía de centro & $\begin{array}{l}\text { No depender de la } \\
\text { inspección masiva }\end{array}$ & $\begin{array}{l}\text { Cultura organiza- } \\
\text { cional para la cali- } \\
\text { dad }\end{array}$ & $\begin{array}{l}\text { Visión de Lide- } \\
\text { razgo }\end{array}$ & $\begin{array}{l}\text { Liderazgo y co- } \\
\text { herencia con los } \\
\text { objetivos }\end{array}$ & $\begin{array}{l}\text { Participación del } \\
\text { personal }\end{array}$ \\
\hline \multirow[t]{2}{*}{ Planificación y gestión } & $\begin{array}{l}\text { Eliminar la prácti- } \\
\text { ca de otorgar con- } \\
\text { tratos de compra } \\
\text { basándose exclu- } \\
\text { sivamente en el } \\
\text { precio }\end{array}$ & $\begin{array}{l}\text { Desarrollo de per- } \\
\text { sonal }\end{array}$ & $\begin{array}{l}\text { Dirección por he- } \\
\text { chos }\end{array}$ & $\begin{array}{ll}\text { Dirección por } & \text { procesos y he- } \\
\text { chos } & \end{array}$ & $\begin{array}{l}\text { Enfoque basado } \\
\text { en procesos }\end{array}$ \\
\hline & $\begin{array}{l}\text { Mejorar de forma } \\
\text { continua el sistema } \\
\text { de producción y de } \\
\text { servicios }\end{array}$ & $\begin{array}{l}\text { Participación de la } \\
\text { comunidad acadé- } \\
\text { mica y trabajo en } \\
\text { equipo }\end{array}$ & $\begin{array}{l}\text { Valoración de los } \\
\text { empleados y so- } \\
\text { cios }\end{array}$ & $\begin{array}{l}\text { Desarrollo e im- } \\
\text { plicación del per- } \\
\text { sonal }\end{array}$ & Mejora \\
\hline Formación continua & $\begin{array}{l}\text { Instituir la capaci- } \\
\text { tación en el trabajo }\end{array}$ & $\begin{array}{l}\text { Enfoque a benefi- } \\
\text { ciarios }\end{array}$ & $\begin{array}{l}\text { Aprendizaje or- } \\
\text { ganizacional y } \\
\text { personal. Mejora } \\
\text { continua }\end{array}$ & $\begin{array}{l}\text { Aprendizaje, in- } \\
\text { novación y mejo- } \\
\text { ra continua }\end{array}$ & $\begin{array}{l}\text { Toma de decisio- } \\
\text { nes basada en la } \\
\text { evidencia }\end{array}$ \\
\hline $\begin{array}{l}\text { Gestión y colaboración } \\
\text { entre instituciones }\end{array}$ & $\begin{array}{l}\text { Enseñar e instituir } \\
\text { liderazgo }\end{array}$ & & $\begin{array}{l}\text { Desarrollo de las } \\
\text { asociaciones }\end{array}$ & $\begin{array}{l}\text { Desarrollo de } \\
\text { alianzas y asocia- } \\
\text { ciones }\end{array}$ & $\begin{array}{l}\text { Gestión de las re- } \\
\text { laciones }\end{array}$ \\
\hline
\end{tabular}




Fomento de la colabo-
ración y del trabajo bien
hecho

Búsqueda de la operatividad

Planificación en la gestión
Calidad organizativa
Potenciar la vocación de
servicio
Formación continua
Líneas de actuación orien-
tadas a la mejora

Desterrar el temor, generar clima para la innovación

Derribar barreras entre las áreas departamentales

Eliminar eslóganes, exhortaciones y metas numéricas para la fuerza laboral

Eliminar estándares de producción y las cuotas numéricas, sustituir por mejora continua

Derribar barreras que impiden el orgullo de hacer bien un trabajo

Instituir un programa vigoroso de educación y reentrenamiento

\section{Emprender accio-} nes para alcanzar la transformación

Nota: elaboración propia.

Por la información expuesta se puede deducir que, para el modelo Deming, es importante crear y difundir la visión, propósito y misión de la empresa o, en este caso, institución educativa. Los principios en Deming se tratan de forma muy concreta y son gestionados gradualmente, paso a paso. En los demás modelos, excepto en el modelo Baldrige, el número de principios es menor, gestionando, por tanto, el proceso de forma más general y menos gradual.

En todos los modelos se persigue la mejora y la búsqueda de la calidad de forma continuada, lo cual coincide con el proceso educativo que exige un proceso evolutivo, sumatorio y global. Igualmente, es fundamental la implicación de todos y cada uno de los trabajadores que participan en el proceso, así como también que estos sigan un proceso de formación y especialización continuada (Maderuelo, 2002). Finalmente, se puede incidir que el principal objetivo, en el que concluyen Calidad Total, Baldrige, EFQM e ISO, es dirigir todos los esfuerzos y estrategias hacia la satisfacción y resultados del cliente. 


\subsection{Unificación de los principios de los modelos de gestión de la calidad selecciona- dos}

Dentro de la primera fase de análisis, se exponen los principios de los modelos, unificándolos desde el punto de vista de la temática, lo que ha supuesto la extracción de un total de 16 elementos (ver Tabla 2), que serán sometidos a valoración en una segunda fase. Se aprecia que los principios del modelo Deming y el Baldrige están presentes en 10 de los 16 principios que se extraen, convirtiéndose en los modelos con mayor información a aportar. Siendo los principios de mayor relevancia aquellos que se orientan a la definición de la identidad del centro, gestión y mejora continua de los procesos y búsqueda de la satisfacción del cliente. Seguidamente, el TQM y el EFQM participan con 6 de los 16 principios. El ISO, con 3 de 16 principios, resulta ser el que menor número de elementos aporta a la unificación.

\section{Tabla 2}

\section{Principios unificados de los modelos de gestión de calidad}

\begin{tabular}{|c|c|c|}
\hline Principios & Modelos & Principios unificados \\
\hline 1. Crear y difundir visión, propósito, misión & Deming & 1. Crear y difundir visión, propósito y misión \\
\hline 1. Enfoque en los resultados y en la creación de valor & TQM & \\
\hline 1. Orientación en los resultados & Baldrige & 2. Buscar la calidad total en los resultados \\
\hline 1. Mejoramiento hacia la calidad total & EFQM & \\
\hline 2. Aprender y adoptar la nueva filosofía & Deming & 3. Aprender y adoptar la nueva filosofía \\
\hline $\begin{array}{l}\text { 2. Liderazgo para la calidad } \\
\text { 2. Liderazgo } \\
\text { 3. Visión de Liderazgo } \\
\text { 3. Liderazgo y coherencia con los objetivos } \\
\text { 7. Enseñar e instituir el liderazgo }\end{array}$ & $\begin{array}{l}\text { Deming } \\
\text { TQM } \\
\text { Baldrige } \\
\text { EFQM }\end{array}$ & $\begin{array}{l}\text { 4. Enseñar e instituir el liderazgo para la mejo- } \\
\text { ra de la calidad y coherencia con los objetivos }\end{array}$ \\
\hline 3. No depender más de la inspección masiva & Deming & 5. No depender de la inspección masiva \\
\hline $\begin{array}{l}\text { 3. Cultura organizacional para la calidad } \\
\text { 7. Desarrollo de las asociaciones } \\
\text { 7. Desarrollo de alianzas y asociaciones }\end{array}$ & $\begin{array}{l}\text { TQM } \\
\text { Baldrige } \\
\text { EFQM }\end{array}$ & $\begin{array}{l}\text { 6. Desarrollar alianzas, asociaciones y cultura } \\
\text { organizacional }\end{array}$ \\
\hline $\begin{array}{l}\text { 3. Participación del personal } \\
\text { 5. Participación de la comunidad académica y trabajo en equipo } \\
\text { 8. Responsabilidad Social y Buen hacer ciudadano } \\
\text { 8. Responsabilidad Social } \\
\text { 9. Derribar las barreras que hay entre las áreas departamentales }\end{array}$ & $\begin{array}{l}\text { Deming } \\
\text { TQM } \\
\text { Baldrige } \\
\text { EFQM }\end{array}$ & $\begin{array}{l}\text { 7. Fomentar la participación, responsabilidad, } \\
\text { comunicación y colaboración del personal }\end{array}$ \\
\hline
\end{tabular}


4. Eliminar la práctica de otorgar contratos de compra basándose exclusivamente en el precio

Deming

\section{Dirección por hechos}

4. Dirección por procesos y hechos

4. Enfoque basado en procesos

7. Gestión de las relaciones

4. Desarrollo de personal

5. Desarrollo e implicación del personal

6. Instituir la capacitación en el trabajo

6. Aprendizaje organizacional y personal y mejora continua

6. Aprendizaje, innovación y mejora continua

8. Desterrar el temor, generar el clima para la innovación

11. Eliminar estándares de producción y las cuotas numéricas, sustituir por mejora continua

Baldrige

EFQM

ISO
8. Eliminar la práctica de otorgar contratos de compra basándose exclusivamente en el precio

12. Derribar las barreras que impiden el orgullo de hacer bien un trabajo

13. Instituir un programa vigoroso de educación y reentrenamiento

5. Mejorar de forma continua y para siempre el sistema de producción y de servicios

6. Toma de decisiones basada en la evidencia

5. Valoración de los empleados y de los socios

10. Eliminar los eslóganes, las exhortaciones y las metas numéricas para la fuerza laboral

5. Mejora

11. Perspectiva en sistemas

6. Enfoque a los beneficiarios

9. Agilidad y respuestas rápidas

10. Enfoque en el futuro

14. Emprender acciones para alcanzar la transformación
Deming

TQM

Baldrige

EFQM
9. Dirigir basándose en hechos y procesos para la toma de decisiones. Gestión de las relaciones

Nota: elaboración propia

Deming

ISO

Deming 12. Valorar a los empleados y socios, evitando Baldrige eslóganes, exhortaciones y metas

Baldrige

ISO

13. Perspectiva organizacional por sistemas orientadas siempre hacia la mejora

14. Enfocar el modelo a los beneficiarios

TQM

15. Trabajar la agilidad y prontas respuestas

Baldrige

16. Enfocar las acciones en el futuro para alcanzar la transformación deseada

11. Mejorar de forma continua y para siempre istema de producción y servicios, tomando decisiones basadas en la evidencia

Deming

Baldrige refleje dicha acción 


\subsection{Fusión de los principios de los modelos de gestión de la calidad seleccionados}

La segunda fase de este trabajo ha supuesto la fusión de los principios derivados de la fase anterior con los que se extraen del I. L. y que inspiran el modelo que se busca para gestionar la calidad de los centros educativos de ideario católico. La Tabla 3 presenta dichos principios que han sido previamente clasificados, estableciendo una serie de dimensiones que los engloban de forma general: liderazgo, búsqueda/enfoque satisfacción de personas/clientes, estrategia/formación/mejora continua, gestión de procesos/política/estrategia, alianzas/recursos humanos/identidad y misión y finalmente desafíos/prospectivas/resultados. (ver Tabla 3).

\section{Tabla 3}

Principios fundamentales del modelo de gestión de calidad para centros educativos de ideario católico

$\begin{array}{ccc}\text { Principios fundamentales } & \text { Principios fundamentales del modelo de } \\ \text { Derivados de los modelos de } & \text { Principios fundamentales de Instru- } & \text { gestión de calidad para centros educativos } \\ \text { gestión de Calidad seleccio- } & \text { mentum laboris } & \text { De ideario Católico } \\ \text { nados } & & \end{array}$
1. Crear y difundir visión, propó- sito, misión
1. Redefinir la identidad
1. Crear y difundir visión, propósito y misión

\section{Buscar la calidad total en los resultados \\ 2. Fortalecer la comunidad educativa y cooperar con las instituciones de la iglesia} 3. Aprender y adoptar la nueva fi-
losofía

4. Enseñar e instituir el liderazgo para la mejora de la calidad y coherencia con los objetivos educando ante la sociedad de la información
2. Buscar la calidad total en los resultados. Fortalecer la comunidad educativa y cooperar con las instituciones de la iglesia.

3. Aprender y adoptar la nueva filosofía. Diálogo permanente entre educador y educando

4. Preparar al alumnado para ser críticos 4. Enseñar e instituir el liderazgo para la mejora de la calidad y coherencia con los objetivos. Preparar al alumnado para ser críticos ante la sociedad de la información

5. No depender de la inspección masiva. Promover una educación integral

6. Desarrollar alianzas, asociaciones y cultura organizacional

6. Motivar y animar a los voluntarios para superar la falta de medios y de recursos

6. Motivar y animar a los voluntarios para superar la falta de medios y de recursos

6. Desarrollar alianzas, asociaciones y cultura organizacional

-

7. Fomentar la participación, res- 7. Promover la formación religiosa de las ponsabilidad, comunicación y co- nuevas generaciones laboración del personal
6. Desarrollar alianzas, asociaciones y cultura organizacional. Motivar y animar a los voluntarios para superar la falta de medios y de recursos

6. Desarrollar alianzas, asociaciones y cultura organizacional. Motivar y animar a los voluntarios para superar la falta de medios y de recursos

7. Fomentar la participación, responsabilidad, comunicación y colaboración del personal. Promover los cursos de religión y ciudadanía responsable para los jóvenes 
Principios fundamentales

DERIVADOS DE LOS MODELOS DE

GESTIÓN DE CALIDAD SELECCIO-

NADOS
Principios fundamentales de InStruMENTUM LABORIS
Principios fundamentales del MOdelo de GESTIÓN DE CALIDAD PARA CENTROS EDUCATIVOS DE IDEARIO CATólico
8. Eliminar la práctica de otorgar contratos de compra basándose exclusivamente en el precio

9. Dirigir basándose en hechos y procesos para la toma de decisiones. Gestión de las relaciones

10. Fomentar el aprendizaje, la innovación y la mejora continua con un buen programa que refleje dicha acción
11. Mejorar de forma continua y para siempre el sistema de producción y servicios, tomando decisiones basadas en la evidencia
8. Promover los cursos de religión y ciudadanía responsable para los jóvenes
8. Eliminar la práctica de otorgar contratos de compra basándose exclusivamente en el precio. Promover los cursos de religión y ciudadanía responsable para los jóvenes

9. Dirigir basándose en hechos y procesos para la toma de decisiones. Gestión de las relaciones. Preparar al alumnado para nuestra sociedad multirreligiosa y multicultural

9. Preparar al alumnado para nuestra sociedad multirreligiosa y multicultural

10. Fomentar el aprendizaje, la innovación y la

10. Formar permanentemente a los docentes mejora continua con un buen programa que refleje dicha acción. Formar permanentemente a los docentes

11. Mejorar de forma continua y para siempre el sistema de producción y servicios, tomando decisiones basadas en la evidencia. Organizar los lugares y recursos para la formación permanente del profesorado

11. Organizar los lugares y recursos para la formación permanente del profesorado

12. Valorar a los empleados y socios, evitando eslóganes, exhortaciones y metas

12. Atender a los desafíos de orden jurídico

12. Valorar a los empleados y socios, evitando eslóganes, exhortaciones y metas. Atender a los desafíos de orden jurídico

13. Perspectiva organizacional por sistemas orientados siempre a la mejora

13. Redefinir la idea de universidad (educación superior católica). (Ítem no válido para etapas preuniversitarias.)

13. Perspectiva organizacional por sistemas orientados siempre a la mejora

14. Atender a los interrogantes derivados

14. Enfocar el modelo a los beneficiarios

15. Trabajar la agilidad y prontas respuestas

de la internacionalización de los estudios universitarios. (Ítem no válido para etapas preuniversitarias.)

14. Enfocar el modelo a los beneficiarios.

Relaciones mutuamente beneficiosas con el proveedor

15. Utilizar los recursos on-line para los estudios universitarios. (Ítem no válido para etapas preuniversitarias.)

16. Orientar y acompañar al alumnado:

16. Enfocar las acciones en el futuro para alcanzar la transformación deseada

\section{universidad, empresa y mundo laboral.} (Ítem no válido para etapas preuniversitarias.)

17. Garantizar la calidad de los sistemas académicos
15. Trabajar la agilidad y prontas respuestas

16. Enfocar las acciones en el futuro para alcanzar la transformación deseada. Orientar y acompañar al alumnado: universidad, empresa y mundo laboral

17. Garantizar la calidad de los sistemas académi$\cos$ 
Principios fundamentales DERIVADOS DE LOS MODELOS DE GESTIÓN DE CALIDAD SELECCIONADOS
Principios fundamentales de InstruMENTUM LABORIS
Principios fundamentales del modelo de GESTIÓN DE CALIDAD PARA CENTROS EDUCATIVOS DE IDEARIO CATÓLICO
18. Analizar la autonomía de las universidades. (Ítem no válido para etapas preuniversitarias.)

19. Preparar el cambio e identidad católica de la universidad. (Ítem no válido para etapas preuniversitarias.)
18. Organizar mediante un sistema de gestión. Concretar y priorizar desafíos

19. Analizar resultados de las posturas tomadas ante los desafíos y mostrar los aspectos positivos y negativos. Líneas prospectadas para el futuro

Nota: elaboración propia

Los principios resultantes se obtienen de los modelos seleccionados refundidos y ampliados. Entre ellos, los principios que atienden a redefinir la idea de universidad, atender a la universalización de los estudios universitarios, utilizar los recursos on-line para los estudios universitarios, orientar y acompañar al alumnado en su trayectoria universidad, empresa y mundo laboral, analizar la autonomía de las universidades y preparar el cambio e identidad católica de la universidad, no se acogen en el modelo objeto de construcción por estar orientados a las enseñanzas universitarias.

\subsection{Análisis y selección de dimensiones de los modelos de gestión de la calidad se- leccionados}

La tercera fase comienza con un análisis de las dimensiones que configuran cada modelo seleccionado, con el objetivo de extraer aquellas que realmente describan la gestión de calidad de los centros educativos objeto de este estudio (ver Tabla 4).

Tabla 4

Dimensiones de los modelos de gestión de calidad seleccionados

\begin{tabular}{|c|c|c|c|c|c|}
\hline Deming & Calidad Total & BALDRIGE & EFQM & ISO 9001:2015 & $\begin{array}{l}\text { INSTRUMENTUM } \\
\text { LABORIS }\end{array}$ \\
\hline $\begin{array}{l}\text { Liderazgo visio- } \\
\text { nario }\end{array}$ & $\begin{array}{l}\text { Principios básicos } \\
\text { para lograr la cali- } \\
\text { dad total }\end{array}$ & Liderazgo & Liderazgo & Enfoque al cliente & Identidad y misión \\
\hline $\begin{array}{l}\text { Cooperación in- } \\
\text { terna y aprendi- } \\
\text { zaje }\end{array}$ & $\begin{array}{l}\text { Modalidades de } \\
\text { mejoramiento }\end{array}$ & $\begin{array}{l}\text { Planificación estra- } \\
\text { tégica }\end{array}$ & Personas & Liderazgo & Sujetos \\
\hline Aprendizaje & $\begin{array}{l}\text { Ciclo de control } \\
\text { para el mejora- } \\
\text { miento }\end{array}$ & $\begin{array}{l}\text { Enfoque en el clien- } \\
\text { te y en el mercado }\end{array}$ & $\begin{array}{l}\text { Política y Estra- } \\
\text { tegia }\end{array}$ & $\begin{array}{l}\text { Participación del } \\
\text { personal }\end{array}$ & Formación \\
\hline
\end{tabular}




\begin{tabular}{|c|c|c|c|c|c|}
\hline Deming & Calidad Total & BALDRIGE & EFQM & ISO 9001:2015 & $\begin{array}{l}\text { INSTRUMENTUM } \\
\text { LABORIS }\end{array}$ \\
\hline $\begin{array}{l}\text { Gestión de pro- } \\
\text { ceso }\end{array}$ & $\begin{array}{l}\text { Actividades para } \\
\text { iniciar un proceso } \\
\text { hacia la calidad to- } \\
\text { tal }\end{array}$ & $\begin{array}{l}\text { Dimensión, Análisis } \\
\text { y Dirección del co- } \\
\text { nocimiento }\end{array}$ & $\begin{array}{l}\text { Alianzas y recur- } \\
\text { sos }\end{array}$ & $\begin{array}{l}\text { Enfoque basado en } \\
\text { procesos }\end{array}$ & $\begin{array}{l}\text { Desafíos y prospec- } \\
\text { tivas }\end{array}$ \\
\hline Mejora continua & & $\begin{array}{l}\text { Enfoque en los re- } \\
\text { cursos humanos }\end{array}$ & Procesos & Mejora & \\
\hline $\begin{array}{l}\text { Satisfacción del } \\
\text { empleado }\end{array}$ & & $\begin{array}{l}\text { Dirección de pro- } \\
\text { cesos }\end{array}$ & $\begin{array}{l}\text { Resultados en los } \\
\text { clientes }\end{array}$ & $\begin{array}{l}\text { Toma de decisiones } \\
\text { basada en la eviden- } \\
\text { cia }\end{array}$ & \\
\hline \multirow[t]{3}{*}{$\begin{array}{l}\text { Satisfacción del } \\
\text { cliente }\end{array}$} & & $\begin{array}{l}\text { Resultados econó- } \\
\text { micos y empresa- } \\
\text { riales }\end{array}$ & $\begin{array}{l}\text { Resultados en las } \\
\text { personas }\end{array}$ & $\begin{array}{l}\text { Gestión de las rela- } \\
\text { ciones }\end{array}$ & \\
\hline & & & $\begin{array}{l}\text { Resultados en la } \\
\text { sociedad }\end{array}$ & & \\
\hline & & & Resultados clave & & \\
\hline
\end{tabular}

Nota: elaboración propia

Resulta significativo observar que la dimensión que hace referencia al liderazgo está presente en cuatro de los seis modelos. Es interesante también la idea de mejora continua para que, una vez alcanzados los objetivos, el proceso continúe de forma indefinida, actualizándose y adaptándose a las necesidades del cliente, del que todos los modelos buscan la satisfacción; en el caso de las instituciones educativas se centrará en el alumnado, familias, profesorado, personal de administración y servicios y, en definitiva, en todas las personas que formen parte de la comunidad educativa. La cooperación interna y el aprendizaje, que nos indica el modelo Deming, incidirá en las dimensiones de personal y formación, remarcando la importancia de la mejora continua. La identidad y misión que queda explícitamente señalada en el Instrumentum Laboris marca el eje principal del modelo que aquí se trata de construir.

Este análisis permite extraer, por lo tanto, cuatro dimensiones que, de todas las expuestas, permiten reconocer de manera integral un sistema de gestión de calidad de centros educativos: liderazgo, organización y gestión, personal y formación.

\subsection{Descripción de las dimensiones y subdimensiones de los modelos de gestión de la calidad seleccionados}

Un segundo momento de análisis en esta tercera fase lo constituye la descripción de estas dimensiones a través de sus elementos constitutivos, que generarán finalmente los indicadores de evaluación para estimar la calidad de las instituciones educativas de ideario católico (ver Tabla 5).

Atendiendo a las especificaciones de cada modelo, se registran las subdimensiones citadas. Con respecto a la dimensión liderazgo, se puede destacar la definición de la identidad, la importancia de atender al cliente, la necesidad de formación constante para todos los profesionales que colaboran y la formación religiosa (ver Tabla 5). 
Tabla 5

Elementos de la dimensión liderazgo
SUBDIMENSIONES
Modelos
DESCRIPCIÓN DE LAS SUBDIMENSIONES

Redefinir identidad y misión

Deming, Baldrige, EFQM, ISO e Instrumentum Laboris

Enfoque al cliente Deming, Baldrige

Tener una misión y visión definidas y actuar como modelo de referencia. Existiendo una coherencia entre lo que se dice, se hace, y el propósito y visión de la organización.

Capacidad para leer las necesidades, expectativas e intereses de los grupos de interés; vender, negociar y estar presente en los círculos de decisión pertinentes.

Fomentar el compromiso y desarrollo profesional de las personas a su cargo.

\begin{tabular}{|c|c|c|}
\hline $\begin{array}{l}\text { Gestión de las rela- } \\
\text { ciones }\end{array}$ & ISO9001: 2015 & Fomentar el compromiso y desarrollo profesional de las personas a su cargo. \\
\hline $\begin{array}{l}\text { Planificación estra- } \\
\text { tégica }\end{array}$ & Baldrige & $\begin{array}{l}\text { Conocer el sistema de gestión, el organigrama y sus funciones para llevar un } \\
\text { control del rendimiento y participación activa. }\end{array}$ \\
\hline Resultados clave & EFQM & Examinar el desempeño de la organización y su mejora en todas las áreas clave. \\
\hline Pastoral juvenil & Instrumentum Laboris & Formación religiosa para jóvenes. \\
\hline Pastoral social & Instrumentum Laboris & $\begin{array}{l}\text { Fortalecimiento de la comunidad educativa y cooperación con las institucio- } \\
\text { nes de la iglesia. }\end{array}$ \\
\hline
\end{tabular}

Nota: elaboración propia.

En cuanto a la dimensión organización y gestión, sus subdimensiones constitutivas derivan de la necesidad de atender al mantenimiento y mejora continua de todas las funciones de la organización, conocer el sistema de medición y procesos para informar sobre los desempeños de la institución anticipándose a las exigencias de los distintos grupos de interés, enunciar planes de acción y concreción de objetivos, lograr la mejora continua y satisfacción del cliente, analizar hechos, pruebas y datos recogidos para lograr objetividad en la toma de decisiones, reconocer el potencial del personal y su gestión y evaluar continuamente todos los procesos. (ver Tabla 6).

\section{Tabla 6}

Elementos de la dimensión organización y gestión

SUBDIMENSIONES

Dimensión, Análisis y Dirección

del conocimiento

Enfoque basado en procesos

Política y Estrategia

\section{Modelos}

Baldrige

ISO 9001:2015

EFQM

\section{DESCRIPCIÓN DE LAS SUBDIMENSIONES}

Mantenimiento y mejora continua de todas las funciones de la organización.

Conocer el sistema de medición para proporcionar información sobre el desempeño del proceso.

Analizar y recoger la información con el fin de prever las exigencias del ámbito de la empresa, anticiparse y conocer las exigencias de sus distintos grupos de interés. 
Actividades para iniciar un proceso hacia la calidad total

Calidad Total

Hacer uso de la política y estrategia para enunciar planes de acción y concreción de objetivos, priorizar objetivos y actividades y analizar el nivel en el que todos los trabajadores entienden este proceso.

\section{Aprendizaje-Formación}

Deming, ISO 9001:2015

Toma de decisiones basada en la evidencia

ISO 9001:2015

Analizar hechos, pruebas y análisis de datos para lograr objetividad y confianza en las decisiones tomadas.

Enfoque en los recursos humanos

Baldridge

Reconocer el potencial del personal y su gestión.

Ciclo de control para el mejoramiento. Modalidades de mejoramiento. Mejora continua

Calidad Total
Evaluar de forma continua el proceso: Diseño, ejecución, medidas de control y ajuste.

Nota: elaboración propia

En cuanto a la dimensión personal, sus subdimensiones surgen para responder a la satisfacción del empleado, del cliente, perseguir la participación del personal, y fortalecer la cooperación interna y el aprendizaje común (ver Tabla 7).

Tabla 7

Elementos de la dimensión personal

\section{SUBDIMENSIONES}

Modelos

\section{DESCRIPCIÓN DE LAS SUBDIMENSIONES}

\begin{tabular}{|c|c|c|}
\hline Satisfacción del empleado & Deming & Se pretende obtener resultados positivos y mayores índices de productividad. \\
\hline Satisfacción del cliente & Deming & Medir cómo el servicio alcanza o sobrepasa las expectativas del cliente. \\
\hline $\begin{array}{l}\text { Participación del personal. } \\
\text { Cooperación interna y } \\
\text { aprendizaje }\end{array}$ & ISO $9001: 2015$ & $\begin{array}{l}\text { El personal, a todos los niveles, es la esencia de una organización, y su total } \\
\text { compromiso posibilita que sus habilidades sean usadas para el beneficio de la } \\
\text { organización. }\end{array}$ \\
\hline
\end{tabular}

Nota: elaboración propia

En cuanto a la formación, para atender a todo el personal implicado en la comunidad educativa, se enfoca hacia directivos, docentes, alumnados y personal de atención y servicios (ver Tabla 8). 
Tabla 8

Elementos de la dimensión formación

\begin{tabular}{|c|c|c|}
\hline SUBDIMENSIONES & Modelos & DESCRIPCIÓN DE LAS SUBDIMENSIONES \\
\hline Formación directivos & $\begin{array}{l}\text { Instrumentum } \\
\text { Laboris }\end{array}$ & $\begin{array}{l}\text { Conocimiento del marco normativo, la organización y gestión de un centro } \\
\text { docente y la gestión de los recursos. }\end{array}$ \\
\hline Formación docentes & $\begin{array}{l}\text { Instrumentum } \\
\text { Laboris }\end{array}$ & $\begin{array}{l}\text { Realización de formación y preparación de formadores. Actualización conti- } \\
\text { nua en pedagogía y metodología. }\end{array}$ \\
\hline Formación alumnado & $\begin{array}{l}\text { Instrumentum } \\
\text { Laboris }\end{array}$ & Ofrecimiento una formación y orientación al alumnado de carácter integral. \\
\hline Formación PAS & $\begin{array}{l}\text { Instrumentum } \\
\text { Laboris }\end{array}$ & $\begin{array}{l}\text { Proporción de formación continua al personal de atención y servicios del } \\
\text { centro educativo. }\end{array}$ \\
\hline
\end{tabular}

Nota: elaboración propia

El resultado de este trabajo es un modelo compuesto por 4 dimensiones, 22 subdimensiones y 80 indicadores (ver Tabla 9).

Tabla 9

Modelo de gestión de calidad de centros de identidad católica

\begin{tabular}{|c|c|c|}
\hline Dimensión & SUBDIMENSIóN & INDICADORES \\
\hline Liderazgo & $\begin{array}{l}\text { Redefinir identidad } \\
\text { y misión }\end{array}$ & $\begin{array}{l}\text { Actualización y centralización del proyecto educativo con los valores del evangelio. } \\
\text { - El centro tiene bien definida su visión y naturaleza católica } \\
\text { - El centro actúa como modelo de referencia de su finalidad católica } \\
\text { - En el centro existe coherencia entre lo que se dice, lo que se hace y el propósito de la } \\
\text { organización } \\
\text { - El profesorado se identifica con el carácter propio del centro }\end{array}$ \\
\hline Liderazgo & Enfoque al cliente & $\begin{array}{l}\text { Cliente satisfecho. } \\
\text { - Las preguntas de esta dimensión coinciden con las de la dimensión personal }\end{array}$ \\
\hline Liderazgo & $\begin{array}{l}\text { Gestión de las } \\
\text { relaciones }\end{array}$ & $\begin{array}{l}\text { Profesionales preparados y comprometidos. } \\
\text { - El profesorado manifiesta su fe y vivencia católica, potenciando la comunicación en } \\
\text { todos los niveles } \\
\text { - El centro proporciona a los jóvenes una experiencia de servicio social, acompañados } \\
\text { por sus profesores o eventualmente por sus padres }\end{array}$ \\
\hline
\end{tabular}


Profesionales que conocen del sistema de gestión y lo llevan a cabo.

- Hay coordinación entre el colegio, la parroquia y la diócesis.
Liderazgo
Planificación estratégica
- El centro planifica teniendo en cuenta los intereses institucionales y su proyecto edu- cativo-evangelizador
- El centro refleja la dinámica emprendedora que le imprime la Diócesis
- El centro promueve la participación de los laicos en el carisma y misión de la Diócesis

$\begin{array}{ll}\text { Análisis continuo del sistema de gestión, análisis de resultados y propuestas de mejora. } \\ \quad & \text { El centro planifica formación pertinente y válida para el personal de administración } \\ & \text { y servicios } \\ \text { Liderazgo } \quad \text { Resultados clave } \quad \text { El centro planifica formación pertinente y válida para las familias } & \\ = & \text { El centro planifica formación pertinente y válida para los docentes } \\ = & \text { El centro cuenta con un plan de acompañamiento en la fe para el personal de admi- } \\ & \text { nistración y servicios }\end{array}$

Formación religiosa de las nuevas generaciones.

Liderazgo Pastoral juvenil

- El centro promueve la formación religiosa de las nuevas generaciones

- El centro cuenta con un plan de acompañamiento en la fe para los estudiantes

- El centro cuenta con un plan de acompañamiento en la fe para las familias

Proyectos compartidos llevados a cabo.

- Desde el punto de vista pastoral, la enseñanza de la religión ocupa un lugar importante en el centro

Liderazgo Pastoral social

- El centro favorece el diálogo fe-cultura e interreligioso

- El centro es inclusivo, acoge a todos sin excluir

- El centro colabora con proyectos que mejoran la sociedad

Mantenimiento y mejora continua de la organización.

- Al seleccionar profesorado y directivos se tiene en cuenta su formación

- Al seleccionar profesorado y directivos se tiene en cuenta su profesionalidad

Dimensión,

Organización Análisis y y gestión Dirección del conocimiento
- Al seleccionar profesorado y directivos se tiene en cuenta la capacitación pedagógica

- Al seleccionar profesorado y directivos se tiene en cuenta su formación pastoral

- Al seleccionar profesorado y directivos se tiene en cuenta su capacidad de gestión y liderazgo

- Al seleccionar profesorado y directivos se tiene en cuenta ser antiguo alumno, tener lazos familiares o ser conocido del centro 
Organización Enfoque basado en y gestión

\section{Organización y gestión}

Uso un diseño de gestión basado en procesos con un sistema de medición que nos proporcione información sobre el desempeño del proceso.

- El Equipo Directivo reconoce y valora el esfuerzo de las personas

- El Equipo Directivo demuestra con su ejemplo, su comportamiento y sus acciones, que defiende y apoya los valores del centro, de tal manera que los refuerza constantemente

- El Equipo Directivo es accesible

- El Equipo Directivo se implica personalmente en desarrollar e implantar en el centro la mejora continua

- El Equipo Directivo fomenta la colaboración y participación de la comunidad educativa en acciones dirigidas a la mejora

Estudio de las opiniones del cliente mediante encuestas y propuestas de mejora continua.

- Las estrategias puestas en marcha son adecuadas para detectar, medir y resolver los conflictos que pudieran plantearse en toda la comunidad educativa

- La elección y funciones de los padres/madres delegados de clase son adecuadas

- En el centro hay líneas prioritarias de actuación que desarrollan programas tendentes a la prevención del fracaso y del abandono escolar

- El centro posee criterios pedagógicos para la asignación de las tutorías

Buena formación del equipo directivo, aplicación firme de la política y estrategia del centro y preparación de todos los trabajadores.

Actividades para

Organización iniciar un proceso y gestión

hacia la calidad total.

- Los objetivos del plan de centro corresponden a las necesidades reales del centro y de su entorno

- Existen medidas y programas para atender al alumnado con necesidades específicas de apoyo educativo

- El centro posee programas de apoyo a la integración que son revisados y evaluados periódicamente

Todos los trabajadores reciben formación continua para la mejora del desempeño de su trabajo.

- La acción tutorial está planificada e integrada en relación con las finalidades del centro

Todas las decisiones se toman basándose en la objetividad comprobada.

Organización y gestión
Toma de decisiones basada en la evidencia.
- El equipo de orientación educativa del centro, planifica y actúa asesorando, cuando es requerido, al alumnado, a las familias, al profesorado

- Los criterios pedagógicos e integradores son prioritarios a la hora de la distribución de grupos 
Excelencia y buenos resultados gracias al trabajo realizado por el personal

- El centro trabaja en red y colabora con otros centros

- El centro promueve la cultura emprendedora

Organización Enfoque en los y gestión
- El centro favorece la participación activa de los estudiantes

- El centro favorece la participación activa de las familias

- El centro favorece la participación activa del profesorado en la planificación y toma de decisiones.

El objetivo principal de la organización es la mejora continua.

Ciclo de

control para el

Organización mejoramiento. y gestión Modalidades de mejoramiento.

Mejora continua.

Satisfacción del empleado.
- El centro acoge y atiende a los estudiantes en situaciones económicas difíciles

- El centro cuenta con la colaboración y participación de los antiguos alumnos y alumnas en el proyecto educativo-evangelizador

- El centro facilita la colaboración efectiva entre el profesorado de una misma etapa

- El centro facilita la colaboración efectiva entre el profesorado de diferentes etapas

- El centro facilita la colaboración efectiva entre el profesorado y las familias
Empleado motivado, comprometido y satisfecho.

- El centro está satisfecho con el aprendizaje escolar del alumnado

- El centro está satisfecho con el ambiente de trabajo del profesorado

- El centro está satisfecho con la atención del personal de administración y servicios

- El centro está satisfecho con la atención del personal de administración y servicios hacia el alumnado

Cliente satisfecho.

- El centro está satisfecho con la atención del profesorado hacia el alumnado

- El centro está satisfecho con la atención del profesorado hacia las familias

- El centro está satisfecho con la atención del Equipo Directivo hacia el alumnado

- El centro está satisfecho con la atención del Equipo Directivo hacia el profesorado

Personal

Satisfacción del cliente.
- El centro está satisfecho con la atención del Equipo Directivo hacia las familias

- El centro está satisfecho en cómo el centro favorece el diálogo fe/cultura e interreligioso

- El centro está satisfecho con el tratamiento que realiza el centro de las quejas y reclamaciones presentadas

- El centro está satisfecho con la respuesta obtenida y la comunicación recibida cuando he presentado una queja o reclamación 


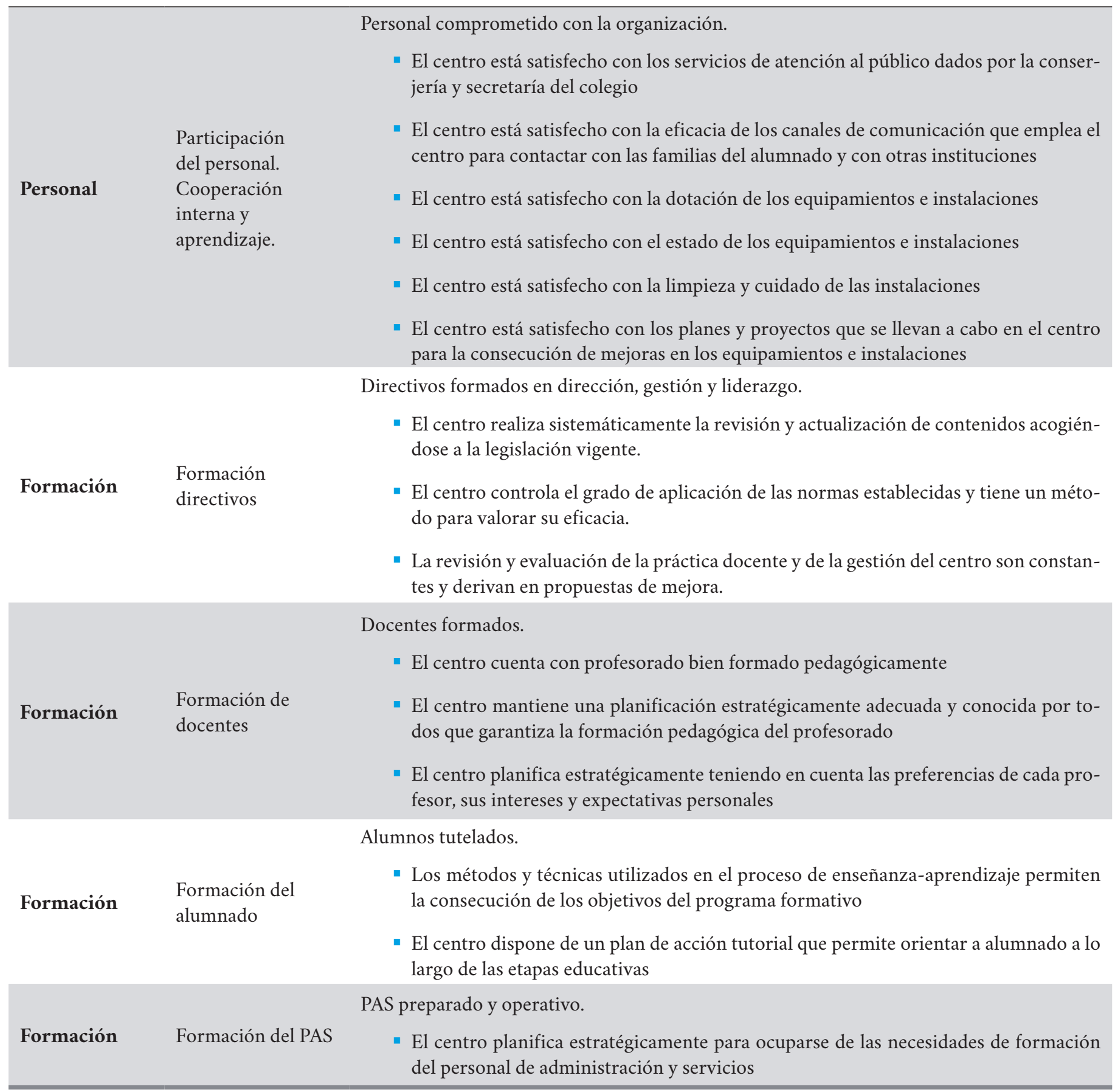

Nota: elaboración propia 


\section{CONCLUSIONES}

Respondiendo al primer objetivo, se han seleccionado los modelos de gestión de la calidad empleados en diferentes organizaciones y sus posibles adaptaciones a instituciones educativas, con la idea de ofrecer una herramienta objetiva que mejore un servicio, con base en una evaluación analítica y diagnóstica y usando criterios igualmente objetivos, cuantificables y medibles como proceso a seguir para determinar si la organización cumple los fines y objetivos establecidos, que oriente de forma fiable la toma de decisiones.

En cuanto al segundo objetivo, analizar y discriminar los principios, dimensiones e indicadores que sirvan de base para la gestión de la calidad de instituciones educativas en los modelos anteriormente seleccionados, se han localizado los indicadores y su definición como claves en esta medición para conseguir una herramienta válida, duradera y concreta aplicable a centros, que imparten enseñanzas obligatorias en un entorno que aporta los valores del Evangelio, como hecho diferencial en el panorama educativo y colabora con las familias en una educación integral de calidad para sus hijos e hijas. Como indica la norma ISO 9001 2015, la organización determina todo lo que necesita para controlar el rendimiento y la eficacia del Sistema de Gestión de Calidad, cómo y cuándo hacer el seguimiento y la medición, además de analizar y evaluar todos los resultados obtenidos. En las Tablas 2 y 4 se encuentran identificados los principios y dimensiones que sirven de base para la gestión de la calidad de instituciones educativas en los modelos anteriormente seleccionados.

Atendiendo al tercer objetivo del estudio, reconocer y determinar los elementos comunes de los diferentes modelos de gestión de calidad y que sirvan de base para la creación de un modelo adaptado a centros educativos de ideario católico tras la revisión bibliográfica de los modelos de gestión de la calidad existentes comparados. El modelo resultante se ha enriquecido con un modelo específico que aporta la visión católica.

El último objetivo planteado, incluir los principios del documento "Educar hoy y mañana. Una pasión que se renueva” (Congregación para la Educación Católica, 2014) en el modelo de gestión de calidad propuesto, para la creación de un modelo aplicable a centros educativos de ideario católico, nos conduce a recopilar información referente a cada elemento y será el paso previo a la construcción de un cuestionario de evaluación que tome como base para su elaboración los diferentes indicadores de calidad seleccionados (Tabla 9), siendo informantes clave los equipos directivos, los equipos docentes y las familias de los centros educativos.

\section{Referencias}

Andrade, C. y Labarca, N. (2011). Fundamentación de los modelos de gestión de la calidad en el servicio de información en instituciones universitarias. Omnia, 17(1), 82-95. Recuperado de http://www.redalyc.org/articulo. oa?id=73718406006

Camisón, C., Cruz, S. y González, T. (2006). Gestión de la calidad: conceptos, enfoques, modelo y sistemas. Madrid: Pearson Educación. Recuperado de https://www.academia.edu/33042332/Gesti\%C3\%B3n_de_la_calidad_ Conceptos_enfoques_modelos_y_sistemas.

Charantimath, P. (2011). Total Quality Management. Singapore: Pearson Educación. Recuperado de https://bit. ly/31TO5Zk 
Congregación para la Educación Católica. (2014). Educar hoy y mañana. Una pasión que se renueva. Recuperado de https://bit.ly/2cpYMcf

Cidad, E. (2004). La gestión de la calidad en las organizaciones de educación superior. Aportación del enfoque de la Organización Internacional de Normalización (ISO). Revista Complutense de Educación, 15(2), 647-686. Recuperado de https://revistas.ucm.es/index.php/RCED/article/view/RCED0404220647A

Del Rincón, D., Latorre, A., Arnal, J. y Sans, A. (1995). Técnicas de investigación en ciencias sociales. Madrid: Dykinson.

Del Río, L. (2008). Cómo implantar y certificar un sistema de gestión de la calidad en la universidad. Investigación en Educación, 5, 5-11. Recuperado de http://reined.webs.uvigo.es/index.php/reined/issue/view/5

Domínguez, G. y Lozano, L. (2005). La calidad, más que una moda, un reto en la Europa de la Sociedad del Conocimiento: la mejora continua más allá de los modelos y las certificaciones (competencias de un formador que aseguran la calidad). Revista Complutense de Educación, 16(1), 57-93. Recuperado de https://revistas.ucm.es/ index.php/RCED/article/view/ RCED0505120057A

Eduardo, L. y Espinoza, O. (2008). Calidad de la educación superior: concepto y modelos. Calidad en la Educación, 28, 248-276. Recuperado de https://calidadenlaeducacion.cl/index.php/rce/article/view/210

Eyssautier, M. (2006). Metodología de la investigación: desarrollo de la inteligencia. México D.F.: Thomson.

Gaviria, J. y Dovale, P. (2014). Propuesta de un modelo de migración de un sistema de gestión de la calidad ISO 9001: 2008 a un sistema de gestión de la calidad basado en la estructura de alto nivel, ISO/DIS 9001: 2015. Medellín: Universidad de Medellín. Recuperado de https://bit.ly/33eIUmY

Gento, S. (1996). Instituciones educativas para la calidad total. Madrid: La Muralla.

González, T. (2000). Evaluación y gestión de la calidad educativa. Un enfoque metodológico. Málaga: Aljibe.

Hasan, A. (2013). Construcción de un modelo de evaluación de la calidad de la enseñanza universitaria desde el punto de vista de los alumnos (Tesis doctoral). Córdoba: Universidad de Córdoba. Recuperado de https://helvia.uco.es/ bitstream/handle/10396/11048/2013000000738.pdf? sequence=1\&isAllowed=y

Hertz, H. (2019). About the Baldrige 2013-2014 Education Criteria for Performance Excellence. [blog]. Recuperado de https://www.nist.gov/blogs/blogrige/thirty-years-evolution-revolutionary-change

Maderuelo, J. A. (2002). Gestión de la calidad total: El modelo EFQM de excelencia. Medifam, 12(10), 41-54. Recuperado de http://scielo.isciii.es/scielo.php?script=sci_ arttext\&pid=S1131-57682002001000004\&lng =es\&tlng=es.

Martínez, C. y Riopérez, N. (2005). El modelo de excelencia en la EFQM y su aplicación para la mejora de la calidad de los centros educativos. Educación XXI, 8, 35-65. Recuperado de https://bit.ly/2VqigVE

Membrado, J. (2002). Innovación y mejora continua según el modelo EFQM de excelencia. Madrid: Díaz de Santos. Recuperado de https://bit.ly/2MnA3c3

Ministerio de Educación y Cultura (M.E.C.). (1997). Modelo Europeo de Gestión de Calidad. Guía para la Autoevaluación. Madrid: Ministerio de Educación y Cultura. 
Municio, P. (2004). La construcción de programas educativos de calidad. Revista Complutense de Educación 15, 485508. Recuperado de https://revistas.ucm.es/index.php/RCED/article/view/RCED0404220485A

Pérez, R., López, F., Peralta, D. y Municio, P. (2000). Hacia una educación de calidad. Gestión, instrumentos y evaluación. Madrid: Narcea.

Rey, R. y Santa María, J. M. (2000). Transformar la educación en un contrato de calidad. Barcelona: Cisspraxis.

Valenzuela, L. y Rosas, J. (2006). Los criterios Baldrige aplicados a la gestión por calidad total y a la excelencia en el desempeño de la educación universitaria. Horizontes empresariales. 6(1), 37-47. Recuperado de http://revistas. ubiobio.cl/index.php/HHEE/article/view/2057

Walton, M. (1993). El método Deming en la práctica; 6 compañias de éxito que usan los principios de control de la calidad total del mundialmente famoso W.E. Deming. Barcelona: Norma.

\section{INFORMACIÓN SOBRE LOS AUTORES}

Inmaculada Díaz Claudel. Profesora superior de instrumento, especialidad de guitarra clásica en el Conservatorio Superior de Música "Rafael Orozco" de Córdoba. Diplomada en Magisterio, especialidad en Educación Musical, por la Universidad de Córdoba. Profesora de educación primaria y secundaria del Colegio Reales Escuelas Pías de la Inmaculada (Córdoba). Su investigación se centra en evaluación de la calidad educativa.

$\bowtie$ inmaculada.diazclaudel@fdemartires.ess

María Amor Martín Fernández. Doctora en Filología Hispánica por la Universidad de Córdoba, Profesora Titular de Escuela Universitaria del área de Didáctica de la Lengua y de la Literatura del Centro de Magisterio Sagrado Corazón, adscrito a la Universidad de Córdoba. Ha publicado libros sobre la poesía española del siglo XV y diversos trabajos relacionados con la creatividad, la formación literaria, la novela española contemporánea, la competencia comunicativa y la innovación docente, que son sus líneas de investigación fundamentales, e imparte cursos sobre creatividad y creatividad literaria. Pertenece al grupo de investigación SEJ049 "Evaluación Educativa e Innovación”, participando en varios proyectos de investigación y en proyectos de innovación docente.

\section{$\triangle$ m.martin@magisteriosc.es}

Ignacio González López. Doctor en Pedagogía por la Universidad de Salamanca. Catedrático del área de Métodos de Investigación y Diagnóstico en Educación de la Universidad de Córdoba. Director del grupo de investigación SEJ049 “Evaluación Educativa e Innovación”, coordinando en varios proyectos de investigación e innovación docente relacionados con la medición y evaluación educativas, y la formación en competencias. 